\title{
Experimental posterior penetrating eye injury in the rabbit. II. Histology of wound, vitreous, and retina
}

\author{
PHILIP E. CLEARY AND STEPHEN J. RYAN \\ From the Department of Ophthalmology, University of Southern California, and Estelle Doheny Eye \\ Foundation, Los Angeles, California, USA
}

SUMMARY The histological findings of the wound, the vitreous, and the retina in the rabbit eye with experimental posterior penetrating injury are described. Wound healing had just begun at 3 days after injury and was well established by 9 to 12 days. It involved proliferation of cells from the episclera and from the choroid. The progression to a fibrous ingrowth from the wound occurred only in eyes with blood in the vitreous. The intravitreal fibroblastic proliferation had begun at 6 days after injury and seemed to be derived from the choroid, the nonpigmented ciliary epithelium and, posteriorly, from the optic nervehead. During the development of retinal detachment the configuration of the peripheral and posterior retina, together with the orientation of vitreous strands, suggested the presence of vitreous traction. We postulate that the presence of contractile fibroblasts (myofibroblasts) in the vitreous may provide the mechanism for vitreous traction. The retinal detachments were also characterised by epiretinal and subretinal membranes, but these were not prominent. The end-stage appearance of a soft, shrunken eye with cyclitic membrane formation and retinal detachment resembles the outcome in many human eyes after severe penetrating injuries.

Severe penetrating injuries in human eyes are characterised histologically by intraocular fibrosis with cyclitic membrane formation and traction retinal detachment (Hogan and Zimmerman, 1962; Coles and Haik, 1972). In fact, traction retinal detachment is the main reason for loss of vision in those injuries which are confined to the posterior segment (Eagling, 1975).

It has been shown in the preceding paper (Cleary and Ryan, 1979) that the experimental model of a posterior penetrating injury in the rabbit eye results in cyclitic membrane formation and in traction retinal detachment. In the present paper we describe the sequence of histological changes.

\section{Materials and methods}

As previously described, rabbit eyes were injured by a standard wound which was $8 \mathrm{~mm}$ long through the pars plana, avoiding the lens and the peripheral retina. Prolapsed vitreous was abscissed and the wound carefully closed by microsurgical techniques with interrupted sutures of $8-0$ silk. After wound

Address for reprints: Mr Philip E. Cleary, FRCS, Ardkeen Hospital, Waterford, Ireland closure the fundus was inspected by indirect ophthalmoscopy and the area of the wound and the peripheral retina examined by scleral indentation. In some eyes autologus blood and in others balanced salt solution was injected through a 25 -gauge needle inserted through the wound and under ophthalmoscopic control into the mid-vitreous, with avoidance of the lens and the retina.

The eyes were enucleated at 3-day intervals for the first 3 weeks after injury as well as at 2 and 3 months after injury. Each group consisted of a minimum of 3 eyes. The eyes were fixed in Bouin's fixative for 24 hours and were then sectioned through the optic nerve and midpoint of the wound for gross examination. Each half eye was examined under the dissecting microscope, and drawings and photographs were made. The eyes were then slowly dehydrated in alcohol and embedded in paraffin.

Vertical serial sections of $5 \mu \mathrm{m}$ thickness were made through the wound, through the retina, and through the optic disc. The sections were stained with haematoxylin-eosin, periodic acid Schiff, and trichrome after Masson.

Controls included 7 normal eyes and 15 eyes with a standard injury but without blood injection. 
Fig. 1 At 3 days after injury. The wound has been artifactually separated during processing (arrow). The striking features are the evidence of proliferation from the episcleral tissues into the wound, and the incarceration of vitreous with vitreous strands radiating from the wound (double arrow) ( $H$ and $E, \times 28)$

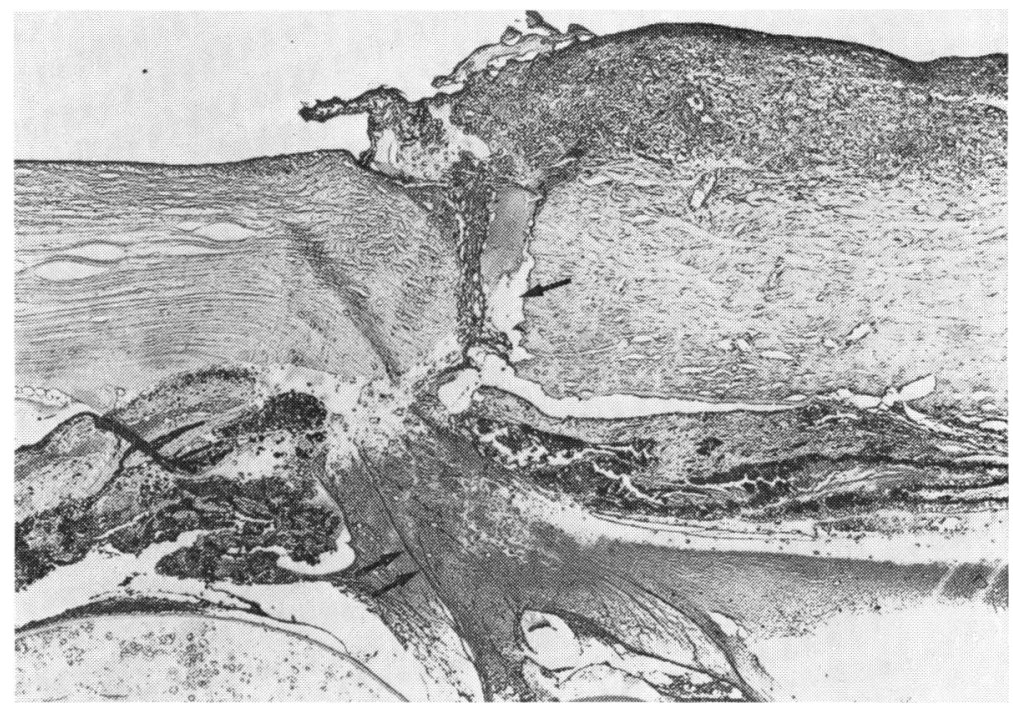

\section{Results}

STANDARD INCISION AND BLOOD INJECTION Early phase (0 to 3 days)

At 3 days after injury the wound and vitreous in the inner aspect of the wound were infiltrated with leucocytes and macrophages (Fig. 1). The edges of the scleral wound remained clearly defined, and there was some early evidence of wound healing with proliferation from the episclera (Fig. 1). Vitreous was incarcerated in the wound, and strands of vitreous radiated from the wound to the posterior retina and also to the peripheral retina in the area of the vitreous base (both directly posterior to the wound and at 180 degrees from the wound). At this stage intravitreal fibroblastic proliferation was not seen.

In these eyes the retina was attached, and the configuration of the peripheral retina resembled that of the normal control eyes and did not suggest the presence of vitreous traction.

\section{Intermediate phase ( 6 to 15 days)}

From 6 to 15 days after injury cellular proliferation occurred and became progressively more prominent at the wound and within the vitreous. In most of these eyes the retina was detached.

During this phase the blood clot became more diffuse and showed signs of absorption, but it remained loculated within the vitreous. The vitreous did not separate posteriorly from the retina. Haemosiderin-containing macrophages were present within the vitreous and in the inner retina.

Wound healing involved proliferation of cells from the episclera initially, but in the inner aspect of the wound fibroblastic proliferation occurred from the uvea and also from proliferation of the nonpigmented ciliary epithelium. Even with good wound apposition fibroblastic ingrowth was already present at 6 days after injury and was prominent by 12 days (Figs. 2 and 3 ). The fibroblasts permeated the blood clot and lined up along the vitreous strands, which were incarcerated in the wound (Figs. 2 and 3). Fibroblastic proliferation into the vitreous also occurred posteriorly from the optic nervehead, where the fibroblasts were also lined up along the vitreous strands (Figs 2 and 3).

During the development of retinal detachment the configuration of the peripheral and posterior retina, together with the orientation of vitreous strands, suggested the presence of vitreous traction (Figs. 4, 5, 6). At 6 days after injury, but more prominently at 9 and 12 days, the peripheral retina assumed a characteristic appearance of being dragged or rolled forward on to the pars plana, not only directly posterior to the wound but also at 180 degrees from the wound (Figs. 5 and 6). In fact, this rolling forward of the peripheral retina was present throughout its entire circumference. Vitreous strands were attached to these areas of peripheral retina, and the vitreous was lined up between the retina and the wound (Figs. 5 and 6). Detachment of the posterior retina occurred typically at 12 days after injury, with a similar appearance of lining up of vitreous strands between the wound and the detached posterior retina.

\section{Late phase ( 18 days to 3 months)}

Eyes examined between 18 days and 3 months after injury showed progression from the initial stages of 


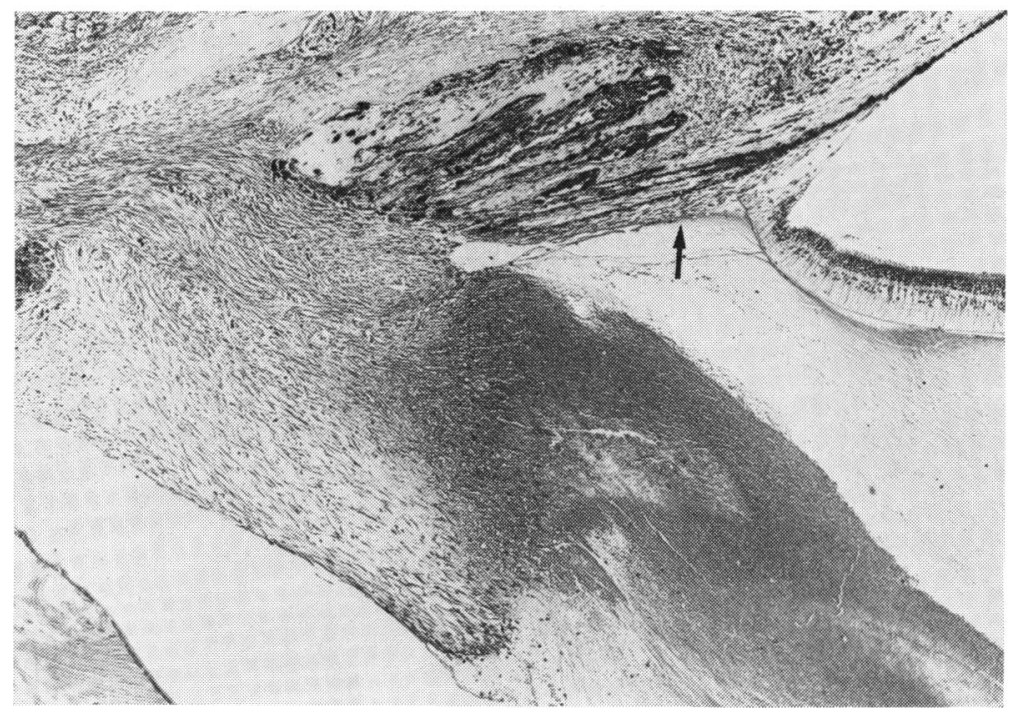

Fig. 2 At 12 days after injury. Note the marked fibrous proliferation extending inward from the wound. The ciliary processes are drawn up to the wound. There is a proliferation of the nonpigmented ciliary epithelium (arrow). The fibroblastic proliferation has invaded the blood clot and is extending posteriorly along the vitreous condensation. In the rabbit eye after injury the vitreous does not separate from the posterior retina, and vitreous becomes condensed and lined up between the wound and the posterior retina. ( $H$ and $E, \times 30)$

Fig. 3 The same eye as Fig. 2 at 12 days after injury. This photograph shows the attachments of the vitreous over the optic nerve head. The blood vessels which are normally present on the surface of the nerve are apparent (arrow). Vitreous strands are attached to the surface of the optic nerve head, and the remarkable feature is the organisation of fibroblasts with their long spindle-shaped nuclei along the vitreous strands (double arrow). ( $H$ and $E, \times 60)$

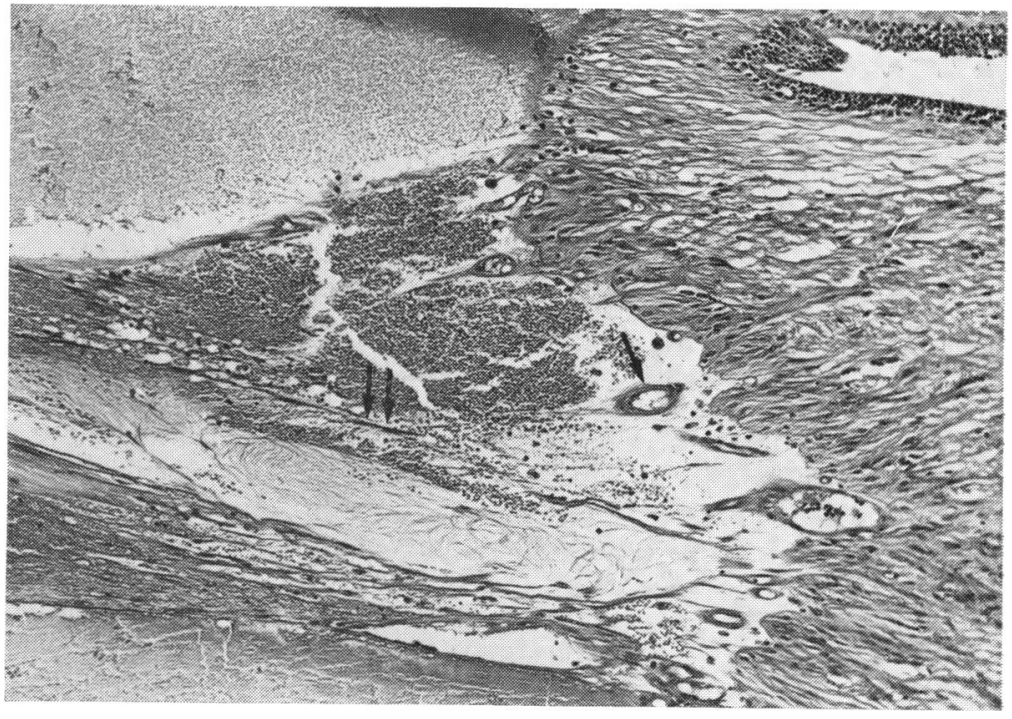

retinal detachment to the advanced stage of total retinal detachment, with the retina drawn up into the wound, with cyclitic membrane formation (Figs. 7 and 8), ciliary body and choroidal detachments, and finally the development of an atrophic shrunken eye.

Proliferations on the surface of the retina with the formation of epiretinal membranes were identified occasionally at 18 and 21 days after injury. However, when the retinal detachments became total and the retina was convoluted and drawn up into the wound, it was not possible to identify discrete epiretinal or subretinal membranes.
Hyperplasia of the retinal pigment epithelium was frequent, with sheets of flattened pigmented cells and plaques of spindle-shaped cells (Fig. 9). In addition, these eyes were characterised histologically by a focal nongranulomatous inflammatory infiltration of the choroid.

\section{STANDARD INCISION AND INJECTION OF BALANCED SALT SOLUTION}

These eyes demonstrated uncomplicated healing of the wound, which had started at 3 days after injury and was well established by 21 days. Proliferation from the episclera appeared as the initial response 
Fig. 4 At 12 days after injury. The fibrous proliferation from the wound is quite prominent. The fibrous ingrowth appears to be derived from the uvea and also from the scleral wound. Note the lining up of the condensed vitreous fibrils which extend from the wound to the posterior retina (arrows). There are a number of inflammatory cells in the vitreous, and overlying the vitreous is the blood clot which is in the process of lysis and organisation. ( $H$ and $E, \times 23$ )
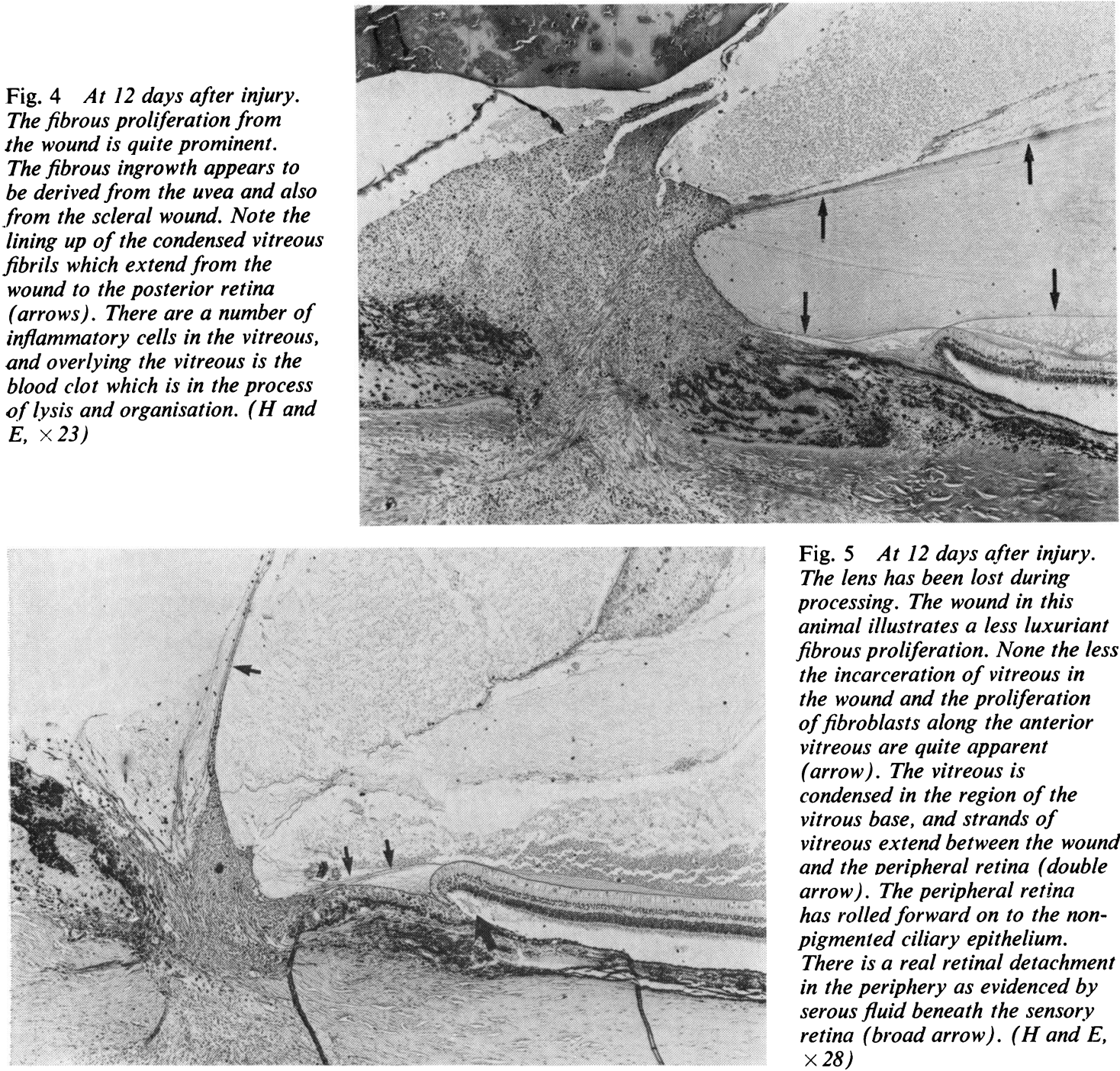

Fig. 5 At 12 days after injury. The lens has been lost during processing. The wound in this animal illustrates a less luxuriant fibrous proliferation. None the less the incarceration of vitreous in the wound and the proliferation of fibroblasts along the anterior vitreous are quite apparent (arrow). The vitreous is condensed in the region of the vitrous base, and strands of vitreous extend between the wound and the peripheral retina (double arrow). The peripheral retina has rolled forward on to the nonpigmented ciliary epithelium. There is a real retinal detachment in the periphery as evidenced by serous fluid beneath the sensory retina (broad arrow). ( $H$ and $E$, $\times 28$ )

Fig. 6 The same eye as Fig. 5 at 12 days after injury. At 180 degrees from the wound the peripheral retina has also rolled forward on to the nonpigmented ciliary epithelium. The condensation of vitreous over the peripheral retina and vitreous base is quite striking. Note again the lining-up of vitreous fibrils and the organisation of inflammatory cells within this matrix. The nonpigmented ciliary epithelium shows a suggestion of early proliferation (arrow). ( $H$ and $E$, $\times .37)$

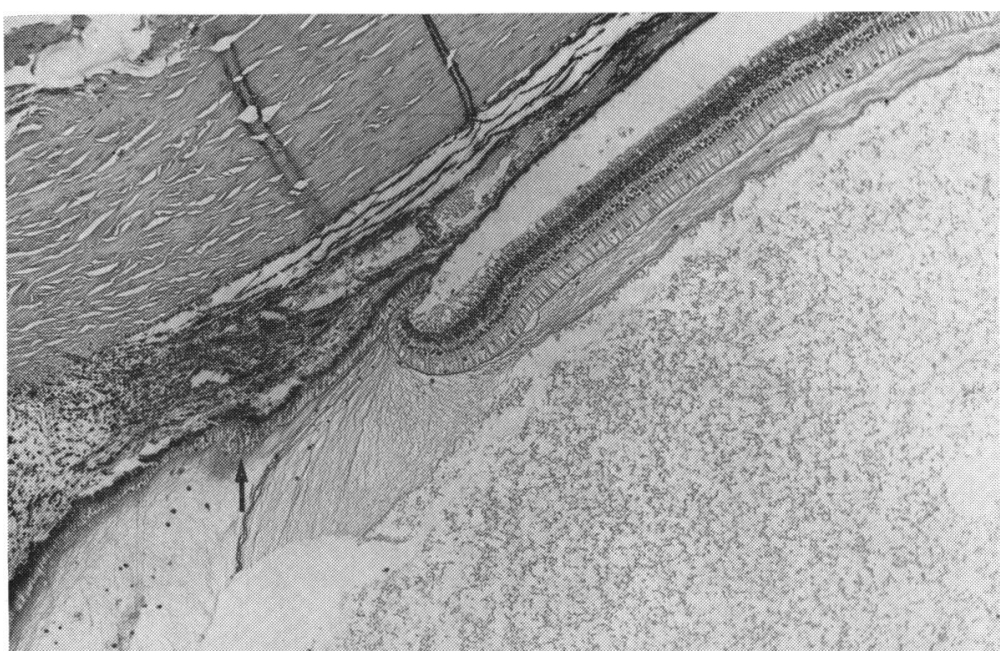




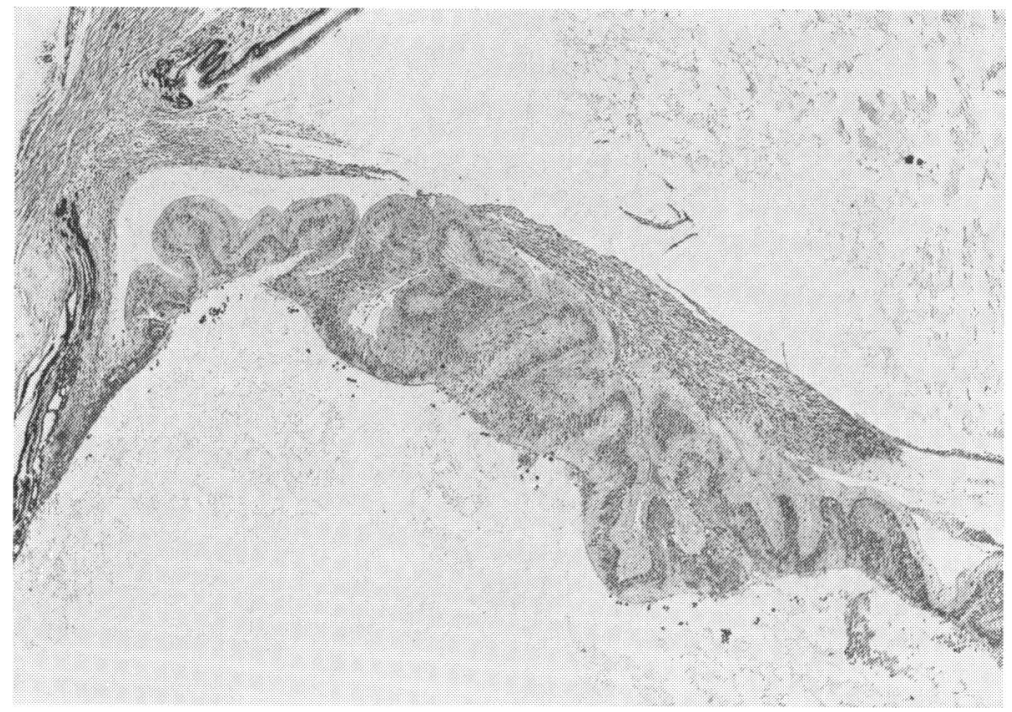

Fig. 7 At 21 days after injury. The lens has been lost during processing. Fibrous proliferation from the wound appears to be derived mainly from the uveal tract. The retina has been thrown into folds and is drawn up to the wound by this fibrous proliferation. The fibrous tissue in the process of forming a cyclitic membrane extends along the surface of the detached retina. ( $H$ and $E, \times 20)$

Fig. 8 Another eye showing the end stage appearance at 4 weeks after injury. The wound is organised and has blood vessels and scattered pigment in the new fibrous tissue (arrows). A marked fibrous proliferation extends inwards from the wound, and swirls of fibrous tissue form a plaque on the surface of the retina. In this animal the entire retina is drawn up into the region of the wound and fibrous proliferation. ( $H$ and $E, \times 56)$

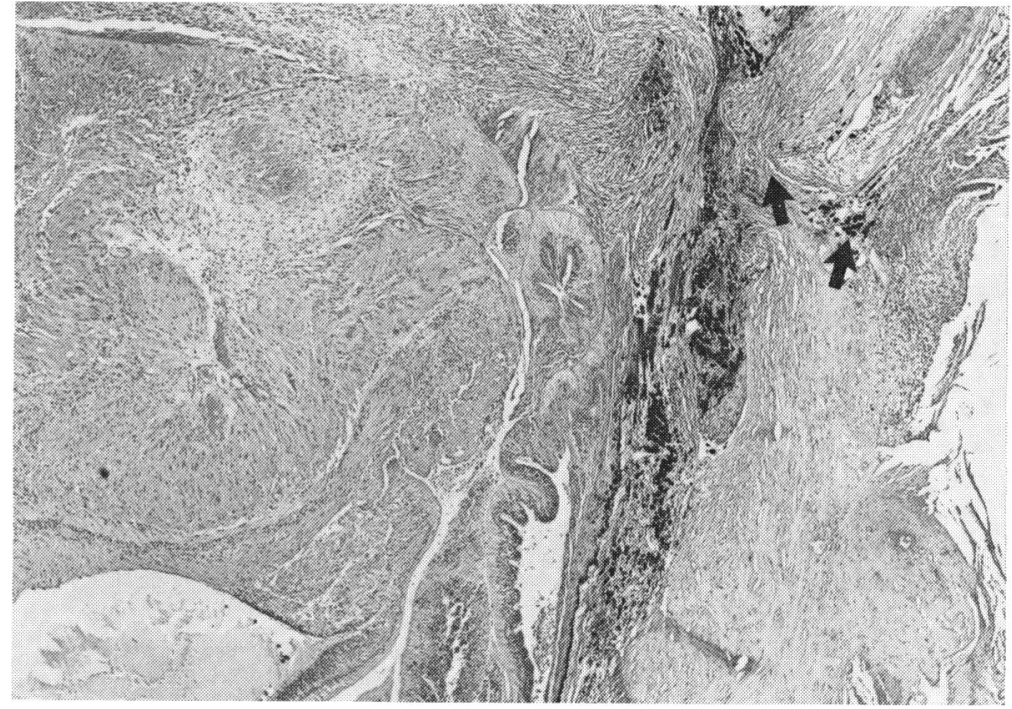

in wound healing, but fibroblastic proliferation from the choroid, together with proliferation of the nonpigmented ciliary epithelium, was also present. Some cellular proliferation occurred on the inner vitreal aspect of the wound, but it did not show progression, and fibroblastic proliferation within the vitreous was not seen (Fig. 10).

Vitreous strands were incarcerated in the wound, and they were also attached to the posterior retina and to the peripheral retina in the region of the vitreous base. The configuration of the retina, however, did not suggest evidence of vitreous traction (Fig. 10).
Eyes examined at 3 months after injury showed a healed and remodeled scar, an attached retina, and the absence of fibrous ingrowth. Some eyes showed proliferation of the nonpigmented ciliary epithelium just posterior to the wound.

\section{STANDARD INCISION WITH INJURY TO THE RETINA}

Typically, wound healing was uncomplicated, and, although vitreous was incarcerated in the wound, neither a fibrous ingrowth from the wound nor intravitreal fibrous proliferations were seen.

The detached retina showed the usual associated 
Fig. 9 At 2 months after injury. Hyperplasia of the retinal pigment epithelium has occurred. Note the tubular proliferation of the retinal pigment epithelium cells (arrow). Note the overlying plaque of partially depigmented spindle cells (double arrows), presumably laid down by the pigment epithelium during its proliferation into the subretinal space. $(H$ and $E, \times 22)$
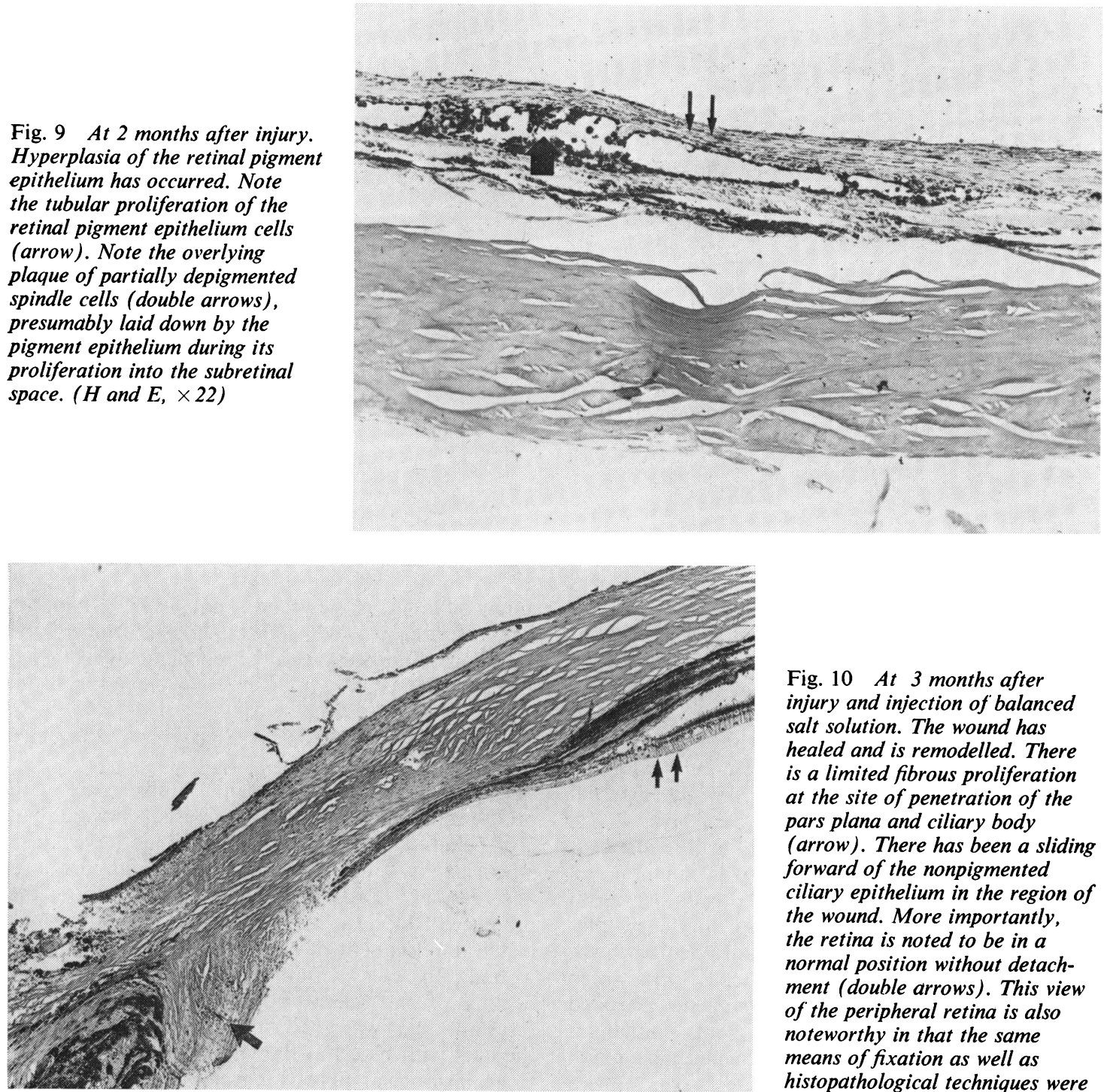

Fig. 10 At 3 months after injury and injection of balanced salt solution. The wound has healed and is remodelled. There is a limited fibrous proliferation at the site of penetration of the pars plana and ciliary body (arrow). There has been a sliding forward of the nonpigmented ciliary epithelium in the region of the wound. More importantly, the retina is noted to be in a normal position without detachment (double arrows). This view of the peripheral retina is also noteworthy in that the same means of fixation as well as histopathological techniques were employed as for all other eyes.

Thus we can feel confident that the rolling forward of the peripheral retina on to the pars plana ciliary epithelium observed in eyes with a standard injury and blood injection is not an artifact due to processing. $(H$ and $E, \times 26)$

degenerative changes, with loss of photoreceptors and intraretinal cystoid swelling. In some areas the retina was thrown into folds, which could be related to the presence of epiretinal membranes. However, the configuration of the retinal detachment remained funnel shaped, and the retina was not drawn up into the wound.

As early as 21 days after injury epiretinal membranes were found, which in some eyes were connected to the underlying retina by a small bridge of tissue and which contained cells with abundant cytoplasm and oval-to-spindle-shaped nuclei. In other eyes the epiretinal membranes may have originated from the prepapillary blood vessels (Fig. 11). The epiretinal membranes also contained pigmented cells which stained positive with Prussian blue for iron. These cells had the appearance of haemosiderin-laden macrophages.

Subretinal membranes lying outside the external limiting membrane could also be found as early as 


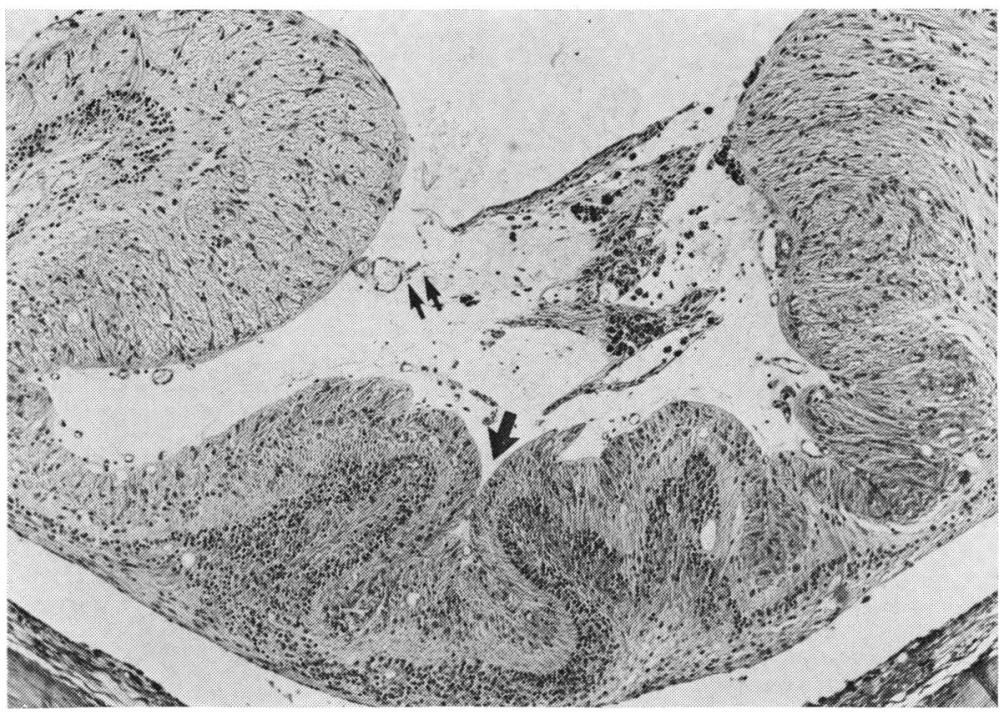

Fig. 11 At 2 months after injury. Full-thickness retinal folds are present. The medullated and nerve fibre layers have been pulled close to each other and approximated in one region (arrow). The epiretinal membrane consists of cells with abundant cytoplasm and oval nuclei which may be glial in origin, and also contains pigmented haemosiderinladen macrophages. In addition the epiretinal membrane has continuity with the blood vessels which are normally present on the surface of the medullary rays in the rabbit eye (double arrows). ( $H$ and $E, \times 45$ )

21 days after injury, but were very uncommon and were confined to retinal folds (Fig. 12). Within retinal folds it was more common to observe a proliferation in the outer retina, of irregularly arranged cells with abundant cytoplasm and oval or spindle-shaped nuclei, but lying within the external limiting membrane (Fig. 12). These cells had displaced the outer retinal layers from the external limiting membrane to the inner nuclear layer.

When the retina was detached, proliferation of the retinal pigment epithelial cells was common and was observed as early as 15 days after injury. Both diffuse hyperplasia with sheets of flattened pigmented cells and plaques of partially depigmented, closely packed, spindle-shaped cells were seen (Fig. 9). When the retinal detachment extended anteriorly to the ora, the pigment epithelium proliferated in the area of the detached pars plana epithelium, becoming 2 or 3 layers thick with irregularly arranged depigmented cells.

Two additional eyes that suffered spontaneous choroidal and subretinal haemorrhage during the injury were enucleated immediately after wound closure. These eyes had a choroidal vascular bed that was engorged with blood.

\section{Discussion}

In experimental posterior penetrating injury in the rabbit eye we observed intraocular fibrous proliferation, the formation of cyclitic membranes, and the development of traction retinal detachment. Human eyes suffer the same sequelae after severe posterior penetrating injuries, although the time course is quite different (Coles and Haik, 1972; Eagling, 1975; Winthrop et al., 1978). The evolution of the pathological process can be conveniently divided into 2 stages.

(1) FIBROUS INGROWTH AND INTRAVITREAL FIBROUS PROLIFERATION

The process of wound healing and repair with proliferation from the episclera and choroid had just begun at 3 days after injury and was well advanced by 9 days, which accords with previous observations (Duke-Elder, 1965). However, the presence of blood in the vitreous had a major effect in the development of a fibrous ingrowth from the wound. In eyes without blood in the vitreous healing of the scleral wound was rapid and the resultant scar was inconspicuous. By contrast, in the eyes with blood in the vitreous, the progression to an intraocular fibrous ingrowth from the wound was a constant feature. Other factors are recognised as important in the development of a fibrous ingrowth, such as incarceration of tissues and poor wound apposition (Duke-Elder, 1965). Yet in these experiments despite good wound apposition a prominent fibrous ingrowth was typical.

During intravitreal fibrous proliferation the cells appeared to line up along vitreous strands as a scaffolding. Cellular proliferation into the vitreous from the wound appeared to be derived not only from the episclera and choroid, but also from the non-pigmented ciliary epithelium (Fig. 14). These cells hypertrophy and become elongated and may proliferate along the vitreous strands to which they are attached. The normal nonpigmented ciliary 
Fig. 12 At 3 months after injury. Note the external limiting membrane delimits the outer retina. An intraretinal glial proliferation has occurred, containing irregularly arranged cells with abundant cytoplasm and oval-to-spindle-shaped nuclei. Inflammatory cells, macrophages, and polymorphonuclear leucocytes are present in the subretinal space. ( $H$ and $E, \times 123$ )
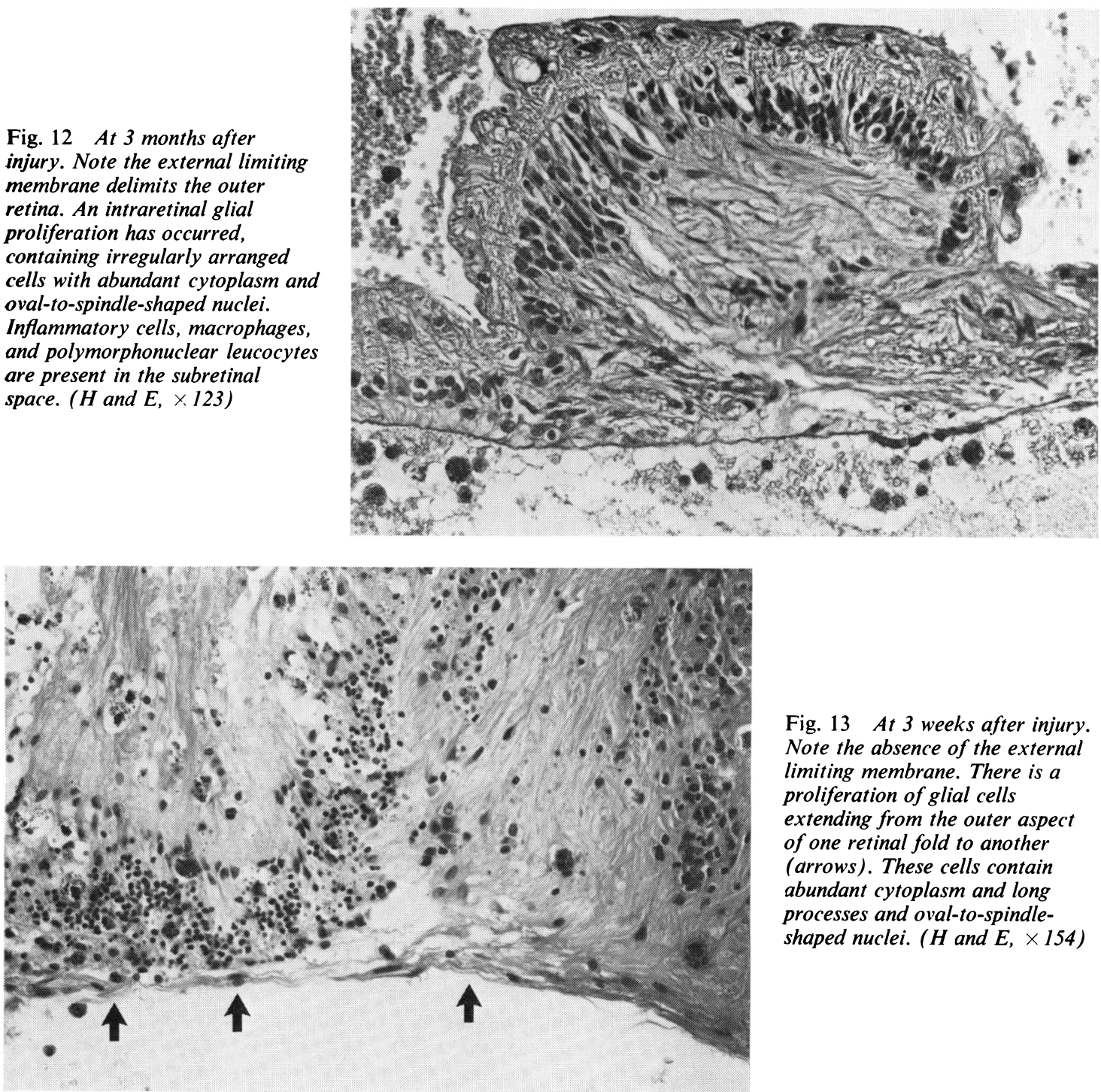

Fig. 13 At 3 weeks after injury. Note the absence of the external limiting membrane. There is a proliferation of glial cells extending from the outer aspect of one retinal fold to another (arrows). These cells contain abundant cytoplasm and long processes and oval-to-spindleshaped nuclei. ( $H$ and $E, \times 154)$

epithelium may give rise to collagen fibrils in the vitreous (Fine and Zimmerman, 1963), and hyperplasia of these cells is recognised in a variety of conditions, including injury and inflammation (Hogan and Zimmerman, 1962).

Intravitreal fibrous proliferation from the optic nervehead has been observed previously in rabbit eyes after injection of autogenous blood or saccharated iron oxide (Cibis and Yamashita, 1959). This proliferation may be derived from epipapillary glial tissue or, more likely, from the prepapillary blood vessels.

The eyes which contained blood also contained monocytes and macrophages; and it is possible that fibroblasts may be derived from haematogenous cells, particularly monocytes and macrophages (Allgower and Hullinger, 1960), although most of the experimental evidence now supports the view that fibroblasts involved in wound healing are derived locally (Grillo, 1964). It is also possible that cells which originated in the pigment epithelium in the area of the wound may have contributed to the fibrous ingrowth, and we observed some spindleshaped cells with pigment other than haemosiderin in their cytoplasm. Certainly, 'fibrous metaplasia' of pigment epithelium is not uncommon (Hogan and 


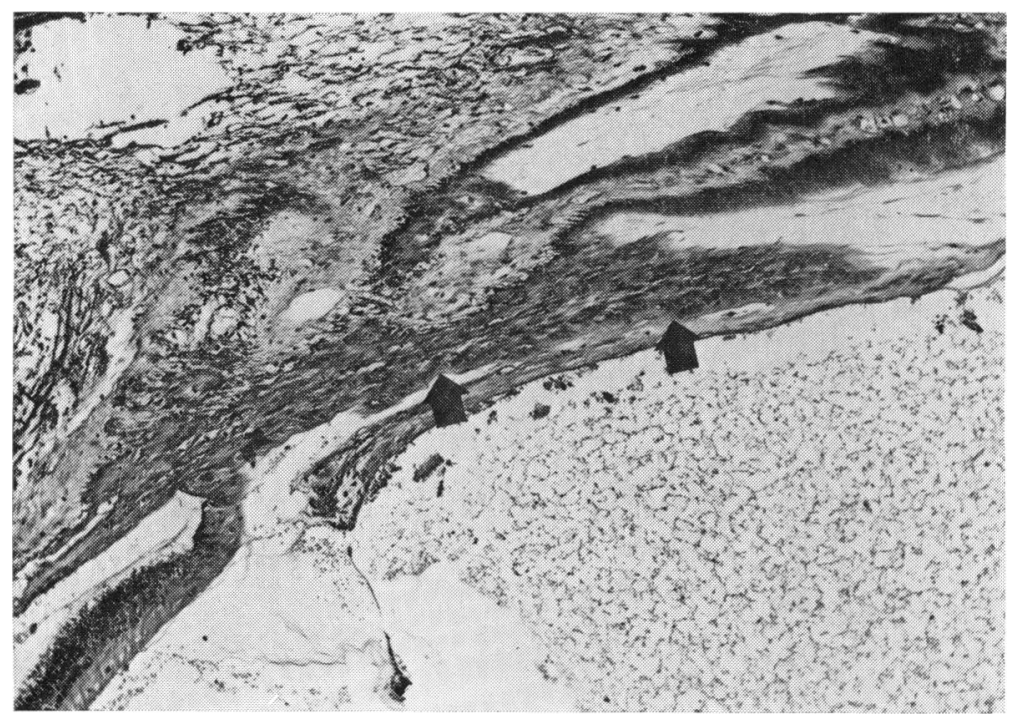

Fig. 14 At 21 days after injury the appearance of the ciliary body at 180 degrees from the wound in an eye with a subtotal retinal detachment. This photograph shows proliferation of the nonpigmented ciliary epithelium. The appearance cannot be explained on the basis of tangential cutting alone, which is apparent in 1 ciliary process. The number of cells and their nuclei suggest a real proliferation of the nonpigmented ciliary epithelium cells. $(H$ and $E, \times 50)$

Zimmerman, 1962; Freyer, 1966; Wallow and Tso, 1972; Tso, 1973; Machemer and Laqua, 1975), but recognition of the epithelial characteristics of these 'fibrocyte-like' cells can be demonstrated only by electron microscopy (Wallow and Tso, 1972; Machemer and Laqua, 1975).

(2) TRACTION RETINAL DETACHMENT In the rabbit eyes, with experimental posterior penetrating injury and blood in the vitreous, we have suggested that the development of retinal detachment may be related to vitreous traction. We interpreted the characteristic configuration of the peripheral retina and elevation of the posterior retina, which was confined initially to the medullary rays, as evidence of vitreoretinal traction (Figs. 4, $5,6)$. The lining up of vitreous strands between the wound and posterior retina and between the wound and peripheral retina in the region of the vitreous base suggests a mechanism by which traction is effected. Certainly in the rabbit eye it is apparent during vitrectomy that the vitreous is tightly attached not only over the peripheral retina in the vitreous base but also over the posterior retina in the region of the medullary rays.

The onset of traction retinal detachment at about 12 days after injury coincides with the presence of significant intravitreal fibroblastic proliferation. In fact fibrous proliferation within the vitreous was present at 6 days after injury but showed a significant progression by 9 to 12 days. Although we have implicated vitreous traction in the development of retinal detachment, the mechanism for traction is obscure but is perhaps related to changes in vitreous collagen, such as reorientation of collagen fibrils as a result of changes in cross-linkage between the native vitreous collagen and the new collagen laid down by fibroblasts.

On the other hand it has been shown that wound contraction occurs independently of collagen formation (Abercrombie et al., 1956) and is mediated by fibroblasts with characteristics typical of smooth muscle cells-myofibroblasts (Gabbiani et al., 1972). The presence of such contractile fibroblasts in the vitreous could provide a mechanism for vitreous traction. Furthermore, the development of vitreous traction leading to traction retinal detachment in the rabbit eye at about 12 days after injury coincides with the rapid increase in the percentage of myofibroblasts observed at between 1 and 2 weeks in experimental full-thickness skin wounds (Rudolph et al., 1977). Although we have not performed electron microscopy on the injured rabbit eyes, we have observed typical myofibroblasts in rhesus monkey eyes after an experimental posterior penetrating injury.

Epiretinal and subretinal membrane formation complicates many posterior penetrating injuries in human eyes (Winthrop et al., 1978). In some contraction of epiretinal membranes at the macula results in macular pucker, while in others massive periretinal proliferation leads to inoperable retinal detachment (Hutton et al., 1976; Benson and Machemer, 1976; Faulborn et al., 1977; Winthrop et al., 1978). In the rabbit eye epiretinal membranes were not common and were probably glial in origin, as indicated by the bridges connecting the epiretinal fibrous tissue and the retina. In human eyes the 
development of epiretinal fibrosis and surface wrinkling retinopathy has been linked with detachment of the posterior vitreous (Roth and Foos, 1971; Wise, 1975; Gass, 1977), though the relationship is far from clear (Foos, 1977). It is of interest, therefore, that in the rabbit eye posterior vitreous detachment does not occur, and we have commented on the tight attachment between the vitreous and posterior retina over the medullary rays.

It is recognised that subretinal membrane in both human and monkey eyes can have their origin in the retinal pigment epithelium (Machemer and Laqua, 1975; Machemer et al., 1978). In the rabbit we noted that proliferation of pigment epithelial cells was common in eyes in which the retina was detached. Nonetheless we found subretinal membranes infrequently in the rabbit eyes, while their appearance and continuity with the retina and a total lack of pigmentation suggested a glial cell rather than pigment epithelium cell origin.

The rabbit eyes enucleated at 3 months after a standard injury and blood injection were soft, the anterior chamber was frequently absent, the lens was often cataractous, the retina was detached, and a cyclitic membrane was present. This end-stage appearance resembles the atrophy and shrinkage seen in many human eyes enucleated after severe ocular trauma (Hogan and Zimmerman, 1962).

We thank Ms K. Borkowski for technical assistance for this paper.

\section{References}

Abercrombie, M., Flint, M. H., and James, D. W. (1956). Wound contraction in relation to collagen formation in scorbutic guinea pigs. Journal of Embryology and Experimental Morphology, 4, 167-175.

Allgower, M., and Hullinger, L. (1960). Origin of fibroblasts from mononuclear blood cells: A study of in vitro formation of the collagen precursor, hydroxproline, in buffy-coat cultures. Surgery, 47, 603-610.

Benson, W. E., and Machemer, R. (1976). Severe perforating injuries treated with pars plana vitrectomy. American Journal of Ophthalmology, 76, 728-732.

Cibis, D., and Yamashita, T. (1959). Experimental aspects of ocular siderosis and hemosiderosis. American Journal of Ophthalmology, 48, 465-480.

Cleary, P. E., and Ryan, S. J. (1979). Experimental posterior penetrating eye injury in the rabbit. I. Method of production and natural history. British Journal of Ophthalmology, 63, 306-311.

Coles, W. H., and Haik, G. M. (1972). Vitrectomy in intra- ocular trauma. Archives of Ophthalmology, 87, 621-628.

Duke-Elder, S. (1965). Diseases of the Outer Eye, Part 2, Vol. 8. Mosby: St Louis, Missouri.

Eagling E. M. (1975). Perforating injuries involving the posterior segment. Transactions of the Ophthalmological Societies of the United Kingdom, 95, 335-339.

Faulborn J., Atkinson A. and Olivier D. (1977). Primary vitrectomy as a preventive surgical procedure in the treatment of severely injured eyes. British Journal of Ophthalmology, 61, 202-208.

Fine B. S., and Zimmerman L. E. (1963). Light and electron microscopic observations on the ciliary epithelium in man and rhesus monkey with particular reference to the vitreous body. Investigative Ophthalmology, 2, 105137.

Foos R. Y. (1977). Vitreoretinal juncture epiretinal membranes, and vitreous. Investigative Ophthalmology, 16, 416-422.

Freyer, W. C. (1966). Reactivity of the retinal pigment epithelium: an experimental and histopathologic study. Transactions of the American Ophthalmological Society. 64, 586-643.

Gabbiani, G., Hirschel, B. J., Ryan, G. B., Statkov, P. R., and Majno, G. (1972). Granulation tissue as a contractile organ. Journal of Experimental Medicine, 135, 719-734.

Gass, J. D. M. (1977). Stereoscopic Atlas of Macular Diseases. Mosby: St Louis, Missouri.

Grillo, H. C. (1964). Derivation of fibroblasts in the healing wound. Archives of Surgery, 88, 82-88.

Hogan, M. J., and Zimmerman, L. E. (1962). Ophthalmic Pathology: An Atlas and Textbook. Saunders: Philadelphia, Pennsylvania.

Hutton, W. L., Snyder, W. B., and Vaiser, A. (1976). Vitrectomy in the treatment of ocular perforating injuries. American Journal of Ophthalmology, 81, 733-739.

Machemer, R., and Laqua, H. (1975). Pigment epithelium proliferation in retinal detachment (massive periretinal proliferation). American Journal of Ophthalmology, 80, $1-23$.

Machemer, R., Van Horn, D., and Aaberg, T. M. (1978). Pigment epithelial proliferation in human retinal detachment with massive periretinal proliferation. American Journal of Ophthalmology, 85, 181-191.

Roth, A. M., and Foos, R. Y. (1971). Surface wrinkling retinopathy in eyes of enucleated at autopsy. Transactions of the American Academy of Ophthalmology and Otolaryngology, 75, 1047-1058.

Rudolph, R., Guber, S., Suzuki, M., and Woodward, M. (1977). The life cycle of the hyofibroblast. Surgery, Gynecology, and Obstetrics, 145, 1-6.

Tso, M. (1973). Photic maculopathy in rhesus monkeys. Investigative Ophthalmology, 12, 17-34.

Wallow, I., and Tso, M. (1972). Proliferation of the retinal pigment epithelium over malignant choroidal tumors. American Journal of Ophthalmology, 73, 914-926.

Winthrop, S., Cleary, P. E., and Ryan, S. J. (1978). The histopathology of ocular trauma. In preparation.

Wise, G. N. (1975). Clinical features of idiopathic preretinal macular fibrosis. American Journal of Ophthalmology, 79, 349-362. 